\title{
The contribution of cetuximab in the treatment of recurrent and/or metastatic head and neck cancer
}

This article was published in the following Dove Press journal:

Biologics: Targets \& Therapy

24 June 2010

Number of times this article has been viewed

\author{
Mohamedtaki A Tejani \\ Roger B Cohen \\ Ranee Mehra \\ Department of Medical Oncology, \\ Fox Chase Cancer Center, \\ Philadelphia, Pennsylvania, USA
}

\begin{abstract}
Recurrent and/or metastatic squamous cell carcinoma of the head and neck (HNSCC) continues to be a source of significant morbidity and mortality worldwide. Agents that target the epidermal growth factor receptor (EGFR) have demonstrated beneficial effects in this setting. Cetuximab, a monoclonal antibody against the EGFR, improves locoregional control and overall survival when used as a radiation sensitizer in patients with locoregionally advanced HNSCC undergoing definitive radiation therapy with curative intent. Cetuximab is also active as monotherapy in patients whose cancer has progressed on platinum-containing therapy. In the first-line setting for incurable HNSCC, cetuximab added to platinum-based chemotherapy significantly improves overall survival compared with standard chemotherapy alone. These positive results have had a significant impact on the standard of care for advanced HNSCC. In this review, we will discuss the mechanism of action, clinical data and common toxicities that pertain to the use of cetuximab in the treatment of advanced incurable HNSCC.
\end{abstract}

Keywords: cetuximab, squamous cell carcinoma of the head and neck, epidermal growth factor receptor

\section{Introduction}

Squamous cell carcinoma of the head and neck (HNSCC) is diagnosed in over 500,000 patients worldwide each year, accounting for 5\% of all malignancies. ${ }^{1}$ In the United States, the estimated incidence was 45,660 new cases and 11,210 deaths in 2007. The most common primary site is the oral cavity. Risk factors for HNSCC include tobacco and alcohol use, ${ }^{2}$ with increasing evidence for a pathogenic role of the human papillomavirus (HPV) in patients lacking the usual risk factors. ${ }^{3}$

SEER (surveillance, epidemiology and end results) data have shown an increase in the annual incidence of base of tongue and tonsil cancers by $2.1 \%$ and $3.9 \%$ respectively, from 1973 to 2001 among white individuals aged 20 to 44 years, whereas the incidence at other sites has declined. The change has been attributed to the increasing prevalence of human papillomavirus (HPV) infection in developed countries, the practice of oral sex and an increasing number of sexual partners. ${ }^{4}$ Approximately $25 \%$ of all HNSCC are associated with HPV and up to $60 \%$ of oropharynx cancers (particularly those of lingual and palatine tonsils) have been associated with HPV. HPV-related HNSCC tend to be poorly differentiated, have basaloid features and present at a lower T stage. ${ }^{5,6}$ Patients with HPV-related head and neck cancers generally have better treatment response and overall survival. ${ }^{6}$

The main site of treatment failure in HNSCC is locoregional recurrence. With improvements in locoregional treatment and control, failure at distant sites with 
metastatic disease is becoming more prevalent. A minority of patients with locoregional failure can be salvaged with surgery or re-irradiation. Until very recently, systemic therapy options were very limited for patients with recurrent disease who were not candidates for locoregional approaches. A series of landmark studies have established the role of cetuximab, a monoclonal antibody targeting the epidermal growth factor receptor (EGFR), in the treatment of locoregionally advanced and potentially curable HNSCC as well as in patients with incurable recurrent and/or metastatic HNSCC. ${ }^{7}$ This review summarizes current data available regarding the evolving use of cetuximab in the treatment of incurable HNSCC. Other reviews have nicely summarized the role of cetuximab in combination with radiation in the curative treatment of HNSCC. ${ }^{8-10}$

\section{EGFR and HNSCC}

The EGFR is a transmembrane cell surface receptor belonging to the erbB family of type 1 receptor tyrosine kinases, which also includes c-erbB2 (Her2/neu), c-erbB3 and c-erbB4. Ligands for the EGFR include EGF itself, transforming growth factor- $\alpha$ (TGF- $\alpha$ ), amphiregulin, epiregulin, betacellulin and heparin-binding EGF-like growth factor (HB-EGF). ${ }^{11}$ EGF and TGF- $\alpha$, often co-expressed with EGFR, are produced by normal and tumor tissue epithelial cells. Higher levels of these ligands are found in tumor and surrounding stroma compared with normal mucosa. EGFR2 (erbB2 or Her2/neu) has no known natural ligands. Neuregulins (NRG) and heregulin are the ligands for ErbB3 and ErbB4.

Four distinct protein domains comprise the EGFR extracellular ligand-binding region. Domains I and III are leucinerich and provide the binding sites for growth factor ligands. Cooperation between domains I and III is required for high affinity binding of EGF. ${ }^{12}$ Most of the anti-EGFR antibodies in the clinic target domain III and thereby interfere with EGF and other ligand binding. Ligand binding is followed by either homodimerization or heterodimerization with another member of the EGFR tyrosine kinase receptor family. ${ }^{13,14}$ EGFR stimulation results in multiple cellular responses including angiogenesis, proliferation, migration, and resistance to apoptosis. Processes that are activated by EGFR stimulation include signal transduction pathways for PI3K-Akt (related to survival and apoptosis evasion) and Ras-Raf-MEK-MAPK (relating to proliferation). ${ }^{15}$ In addition, there is an interaction between EGFR expression and signal transducers and activators of transcription 3 (eg, STAT3), which play a role in the regulation of transcription of genes involved in cell cycle progression such as Fos, Cyclin-D, CDC25A, c-Myc and Pim1 and the upregulation of antiapoptotic genes such as BCL2. ${ }^{16,17}$

EGFR can also translocate to the nucleus where it activates or represses the production of various effector proteins. One key nuclear function of EGFR is phosphorylation and activation of DNA-dependent protein kinase (DNA-PK), an enzyme that is involved in repair of breaks in doublestranded DNA caused by radiation and chemotherapy. ${ }^{18,19}$ EGFR localizing to the nuclear compartment can also enter transcriptional complexes and bind to the promoter region of cyclin D1 to promote cell cycle progression and increase cellular proliferation. ${ }^{20}$ EGFR inhibition delays the repair of chemotherapy-induced DNA damage via modulation of DNA repair genes such as XRCC1 and ERCC1, which could explain some of the increased activity of cisplatin when combined with EGFR inhibitors such as cetuximab. ${ }^{21-23}$

Elevated EGFR expression detected by immunohistochemistry is present in over $90 \%$ of HNSCC specimens and the level of expression is associated with inferior survival, radioresistance and locoregional failure. ${ }^{24-27}$ Unlike lung adenocarcinomas in which activating mutations of the EGFR create ligand independent pathway activity, ${ }^{28,29}$ such EGFR mutations in HNSCC specimens are distinctly uncommon and therefore do not explain the role of the EGFR in the pathogenesis of HNSCC. ${ }^{30,31}$ Thus, the excess activity of the EGFR and related pathways in HNSCC appears to be mostly ligand-dependent, or due to receptor over-expression. A variant of EGFR has been described - EGFRvIII - which is weakly constitutively active in a ligand-independent manner. This truncated form of EGFR was present in $42 \%$ of HNSCC cells in one series. Cells that harbor this mutant are likely to be less responsive to treatment with cetuximab, since EGFRvIII has a deletion of exons 2 through 7, encompassing the ligand-binding domain and the cetuximab-binding site. Further study of the role of EGFRvIII in the pathogenesis and response to treatment in HNSCC is clearly indicated. ${ }^{32}$

Multiple preclinical studies have shown that EGFR inhibition sensitizes HNSCC to the effects of ionizing radiation. ${ }^{7,33-35}$ Similarly, EGFR inhibition with cetuximab demonstrated synergy in combination with chemotherapy in killing HNSCC cells in xenograft tumor models. In mouse A431 xenografts of HNSCC, the combination of cetuximab and cisplatin led to growth inhibition in excess of that achieved with either agent alone. ${ }^{36}$ Similar enhancement by cetuximab of the activity of cisplatin and 5-FU was observed in another cell line, human tongue SCC-25. ${ }^{37}$ 


\section{Cetuximab}

Cetuximab is a chimeric monoclonal antibody ( $65 \%$ human and $35 \%$ murine) constructed on an immunoglobulin (Ig) G1 framework, which targets an extracellular epitope in the EGFR ligand-binding domain. ${ }^{38}$ Cetuximab blockade of the EGFR results in inhibition of tumor growth, metastasis, DNA damage repair and angiogenesis. ${ }^{39,40}$

Several mechanisms that contribute to the anti-tumor activity of cetuximab have been identified. A major mechanism is interference by cetuximab with the binding of natural ligands to the receptor itself, thereby disrupting EGFR signaling pathways. ${ }^{41}$ Another mechanism involves depletion of the targeted receptors from the cell surface via induction of receptor endocytosis. ${ }^{42}$ Finally, cetuximab's construction on an IgG1 framework potentially allows this agent to mediate antibody-dependent cell-mediated cytotoxicity (ADCC) via recruitment of natural killer cells and macrophages. ${ }^{43,44}$ ADCC is influenced by $\mathrm{Fc} \gamma$ receptor polymorphisms that are known to be clinically relevant in follicular lymphoma and metastatic breast cancer and modulate the treatment response to antibodies such as rituximab and trastuzumab. A polymorphism in Fc gammaR IIIa receptor, Fc gammaR IIIa-158, which is expressed on NK cells and associated with enhanced ADCC, is linked with increased cetuximab activity in HNSCC cells in vitro. ${ }^{45}$ Clinically, variation in Fc $\gamma \mathrm{R}$ polymorphisms in patients with HNSCC treated with cetuximab could potentially account for some portion of the varying response rates that are observed in patients. Additional clinical data will be needed, however, before we can assign a definite role to ADCC in the treatment response to cetuximab in patients with HNSCC.

There are several other EGFR targeting antibodies in varying stages of clinical development. Cetuximab, matuzumab and nimotuzumab are constructed on an IgG1 framework that potentially allows these agents to mediate ADCC via natural killer (NK) cells and macrophages. By contrast, panitumumab, a fully humanized anti-EGFR antibody constructed on an IgG2 framework, will not mediate ADCC. ${ }^{46}$ Panitumumab is currently FDA-approved for use in metastatic colorectal in colorectal cancer, and unlike cetuximab is not associated with infusion related hypersensitivity reactions. IMC-11F8 is a newer fully humanized anti-EGFR IgG1 monoclonal antibody that has been tested in the phase 1 setting ${ }^{47}$ and is now being studied in phase 2 and 3 studies in colorectal and non-small cell lung cancer, respectively. Another novel anti-EGFR antibody, $\mathrm{mAb} 806$, is active against cell lines expressing the mutant variant EGFRvIII as well as cell lines in which wild type
EGFR is over-expressed..$^{48}$ Cetuximab, nimotuzumab, panitumumab and matuzumab all prevent ligand binding via interactions with EGFR domain III, and mAb 806 interferes with receptor function via interactions with domain II. ${ }^{49-52}$ The clinical significance of these molecular interactions of antibody and receptor is unknown and is the subject of ongoing basic research.

\section{Cetuximab in the treatment of locally advanced disease}

In 2006, Cetuximab was approved for use in combination with radiation therapy in patients with locally advanced HNSCC. This followed a pivotal phase III international trial, conducted by Bonner et al, in which 424 patients with locally advanced disease were randomized between definitive radiation and concurrent radiation with cetuximab (given at $400 \mathrm{mg} / \mathrm{m}^{2}$ loading dose followed by $250 \mathrm{mg} / \mathrm{m}^{2}$ weekly for 8 planned doses). ${ }^{7}$ Cetuximab plus radiation improved the duration of loco-regional control from 14.9 to 24.4 months $(P=0.005)$ and improved median survival from 29.3 to 49 months $(P=0.03)$. There was no difference in the rates of distant metastatic disease in the two arms. Notably, the acute toxicities of radiation were not exacerbated by the addition of cetuximab. In a retrospective subset analysis, the hazard ratios favored the addition of cetuximab to the altered fractionation radiation regimens. This landmark study was the first to provide proof of principle data for the activity of cetuximab with radiation in the curative setting.

As cetuximab and cisplatin have different mechanisms of action and non-overlapping toxicity profiles, there has been interest in combining both agents with radiation. An exploratory phase II study from MSKCC enrolled 22 patients with locally advanced HNSCC ( $86 \%$ with stage IV disease) to receive cisplatin ( $100 \mathrm{mg} / \mathrm{m}^{2}$ every 3 weeks) and cetuximab ( $400 \mathrm{mg} / \mathrm{m}^{2}$ followed by $250 \mathrm{mg} / \mathrm{m}^{2}$ weekly) along with definitive radiation..$^{53}$ Three-year overall survival and locoregional control rates were unusually positive at $76 \%$ and $71 \%$ respectively. Adverse events resulted in the premature termination of this trial (including 2 on-study deaths, one from pneumonia and one of unknown cause).

A preliminary safety analysis of ECOG 3303 , a phase II study of 61 patients with locally advanced HNSCC has also been reported. ${ }^{54}$ Enrolled patients received cisplatin $\left(75 \mathrm{mg} / \mathrm{m}^{2}\right.$ every 3 weeks $)$ and cetuximab $\left(400 \mathrm{mg} / \mathrm{m}^{2}\right.$ followed by $250 \mathrm{mg} / \mathrm{m}^{2}$ weekly) along with definitive radiation. In the absence of disease progression or unacceptable toxicity, patients continued weekly maintenance cetuximab for six months. Results indicate expected grade $3 / 4$ toxicities 
of anemia, neutropenia, hypomagnesemia, hyponatremia, rash, fatigue and mucositis along with two late grade 4 toxicities (pharynx pain and laryngeal edema), and one attributable grade 5 event (neutropenic fever). Early efficacy data seem promising with median progression-free survival (PFS) of 15.3 months.

The RTOG 0522 (NCT00265941) study is a large, randomized phase III trial that randomized patients to receive either concurrent accelerated radiation and cisplatin or concurrent accelerated radiation, cisplatin and cetuximab. The data are currently not mature and analysis is ongoing. It is hoped that this study will define the role and feasibility of cetuximab when combined with definitive cisplatin-based chemoradiation.

At this time, it is not known whether cetuximab and radiation is equivalent to cisplatin and radiation and there are unfortunately no prospective trials ongoing or planned to examine this important question. An interesting retrospective institutional analysis from MSKCC compared the outcome of 125 patients who received cisplatin $\left(100 \mathrm{mg} / \mathrm{m}^{2}\right.$ every 3 weeks) with radiation to 50 patients who received cetuximab (400 mg/m² loading dose and $250 \mathrm{mg} / \mathrm{m}^{2}$ weekly) with radiation. ${ }^{55}$ Recognizing that these were two different patient populations, multivariate analysis to address prognostic imbalances was performed. Despite this adjustment, results for local failure (LF), disease-free survival (DFS) and overall survival (OS) all favored the cisplatin arm $(P<0.0001$ for LF and DFS, $P=0.0017$ for OS). Thus, definitive radiation with cisplatin chemotherapy currently remains the treatment of choice for medically fit patients.

Given its established activity when combined with radiation and chemotherapy, there is considerable interest in adding cetuximab to induction chemotherapy regimens. In 2008, Argiris et al reported preliminary safety results of a phase II trial in which 39 patients received induction cisplatin ( $75 \mathrm{mg} / \mathrm{m}^{2}$ every 3 weeks for 3 cycles), docetaxel $\left(75 \mathrm{mg} / \mathrm{m}^{2}\right.$ every 3 weeks for 3 cycles $)$ and cetuximab $\left(400 \mathrm{mg} / \mathrm{m}^{2} \mathrm{load}-\right.$ ing and $250 \mathrm{mg} / \mathrm{m}^{2}$ weekly). ${ }^{56}$ This was followed by radiation with concurrent cisplatin ( $30 \mathrm{mg} / \mathrm{m}^{2}$ weekly) and cetuximab (250 $\mathrm{mg} / \mathrm{m}^{2}$ weekly). Patients subsequently received maintenance cetuximab for six months. Serious toxicities during treatment included grade 3/4 neutropenia, infection, anemia, thrombocytopenia, hypomagnesemia, rash, mucositis and diarrhea. Longer follow-up is needed for determination of loco-regional control and survival endpoints.

Investigators from ECOG conducted a study (ECOG 2303) of induction carboplatin/paclitaxel and cetuximab followed by radiation with carboplatin/paclitaxel and cetuximab. ${ }^{57}$ Six months of maintenance cetuximab was given subsequently. Primary site biopsies were completed after the induction phase and again after the chemoradiation phase with complete pathological responses noted in $65 \%$ and $100 \%$ of samples, respectively.

Investigators from MDACC have also presented data integrating cetuximab in an induction regimen with weekly carboplatin and paclitaxel. ${ }^{58}$ This regimen was followed by risk-based definitive therapy. The results are promising with induction therapy that includes cetuximab resulting in a complete response (CR) rate of $19 \%$ and partial response (PR) of $77 \%$. The 3 -year OS rate of $91 \%$ in this population of patients with regionally metastatic N2/N3 disease was encouraging. Six patients had recurrences, 3 of them with T4 disease. Twelve out of 26 tumors tested were HPV-positive; this particular group did not have any recurrences, and had a significantly greater PFS and OS compared to the HPV-negative patients.

Finally, cetuximab has also been added to the TPF regimen (docetaxel, cisplatin and 5-FU), which was investigated in a randomized phase III trial and found to have superior survival results when compared to the 2-drug combination of cisplatin and 5-FU and induction chemotherapy. ${ }^{59,60} \mathrm{~A}$ phase 1 study from Dana-Farber was completed to determine the MTD (maximum tolerated dose) of 5-FU in the TPF regimen when combined with cetuximab for locally advanced HNSCC. ${ }^{61}$ Nineteen patients were enrolled. There was one dose-limiting toxicity (DLT) of febrile neutropenia, and gastrointestinal toxicity with grade 4 enteritis was a serious DLT necessitating reduction of the 5 -FU dose to $850 \mathrm{mg} / \mathrm{m}^{2}$ to lessen toxicity.

A phase II trial from Spain enrolled 50 patients who received induction chemotherapy with cetuximab $\left(400 \mathrm{mg} / \mathrm{m}^{2}\right.$ loading and $250 \mathrm{mg} / \mathrm{m}^{2}$ weekly) along with TPF (docetaxel $75 \mathrm{mg} / \mathrm{m}^{2}$, cisplatin $75 \mathrm{mg} / \mathrm{m}^{2}$ and continuous 5-FU $750 \mathrm{mg} / \mathrm{m}^{2}$ on days 1 to 5 every 3 weeks for 4 cycles). ${ }^{62}$ This was followed by concurrent radiation with weekly cetuximab. There was a high ORR of $78 \%$ after 4 cycles of induction therapy. Grade 3/4 toxicity was significant with neutropenia (24\%), neutropenic fever $(20 \%)$, diarrhea $(12 \%)$, infection $(6 \%)$, thrombocytopenia (4\%), hepatotoxicity (4\%) and hypomagnesemia $(2 \%)$. There were also 2 treatment-related deaths (febrile neutropenia and hepatic insufficiency).

It remains to be proven whether induction chemotherapy followed by chemoradiation is better than chemoradiation alone. However, preliminary data suggest that it is feasible to combine cetuximab with other agents in our efforts to tailor an ideal induction therapy regimen for patients with locally 
advanced HNSCC. Larger studies are required to study if there is any survival benefit to intensifying induction regimens with the addition of cetuximab in both HPV-positive and negative patients.

\section{Treatment of recurrent or metastatic disease}

Treatment options are still too few for patients with locally recurrent or metastatic incurable HNSCC. A limited number of patients with recurrent disease are candidates for salvage therapies, including potentially curative surgery. ${ }^{63}$ Some patients in this setting appear to benefit from re-irradiation although the morbidities of this approach can be substantial and careful patient selection is key. ${ }^{64}$ Chemotherapy therefore remains the mainstay of treatment for the majority of patients with incurable HNSCC.

Prior to the introduction of cetuximab, the most common regimens for recurrent/metastatic HNSCC included cisplatin plus 5-FU, cisplatin plus a taxane or single-agent methotrexate. ${ }^{65-67}$ Bleomycin is also approved in this setting but is not a component of contemporary regimens. Response rates are higher for combination regimens vs monotherapies and regimens containing cisplatin are the most active. Nonetheless, over a period of 30 years median survival with chemotherapy for this population had remained around 6 to 7 months with no clear evidence that any variations in the chemotherapy regimens, including the introduction of newer agents such as taxanes, had any significant additive effects on overall survival. The situation is even worse in patients failing first-line chemotherapy, especially those patients who are demonstrably platinum-resistant. Second-line treatment options for incurable platinum-resistant HNSCC were until recently practically non-existent. ${ }^{68}$

\section{Cetuximab in the first-line treatment of patients with incurable HNSCC}

Burtness and co-investigators in ECOG completed the first clinical trial (E5397) in 2005 investigating the role of cetuximab in the first-line treatment of incurable advanced HNSCC. ${ }^{69} 117$ patients who had not received prior chemotherapy for recurrent and/or metastatic disease were randomized to either cisplatin $\left(100 \mathrm{mg} / \mathrm{m}^{2}\right.$ every 4 weeks $)$ with placebo or to cisplatin with cetuximab $\left(400 \mathrm{mg} / \mathrm{m}^{2}\right.$ loading dose followed by $250 \mathrm{mg} / \mathrm{m}^{2}$ weekly). There was a statistically significant improvement in response rate from $10 \%$ to $26 \%$ with the addition of cetuximab $(P=0.03)$ with a trend towards an improvement in overall survival from 8 to 9.2 months. The 1 -year survival rates for the cisplatin plus cetuximab and cisplatin plus placebo arms were $39 \%$ and $32 \%$ respectively with corresponding 2 -year survival rates of $16 \%$ and $9 \%$. However, the difference in survival was not statistically significant, likely due to a lack of power to detect significant survival differences, as well as the study design, which allowed cross-over to cetuximab of patients who had progressed on the placebo arm. In a phase I/II study performed by Bourhis et al, 53 patients with advanced HNSCC were assigned to treatment in the first-line setting with cetuximab plus platinum plus 5 -FU. ${ }^{70}$ Treatment with this triplet showed an overall response rate of $36 \%$ with a promising median survival of 9.76 months.

In a later and much larger phase III study conducted in Europe, known as the EXTREME trial, 442 patients with advanced HNSCC were randomized to either a platinum-containing doublet or a similar doublet with cetuximab. ${ }^{71}$ Patients had not received any prior treatment for recurrent/metastatic disease. The chemotherapy regimen used was platinum (cisplatin at $100 \mathrm{mg} / \mathrm{m}^{2}$ or carboplatin AUC 5 on day 1) in combination with 5-FU $1000 \mathrm{mg} / \mathrm{m}^{2}$ on days 1 to 4 for a maximum of 6 cycles. Patients randomized to receive cetuximab with chemotherapy could continue to receive maintenance cetuximab until progression. It is important to note that cross-over to cetuximab for those patients initially randomized to chemotherapy alone was not allowed in this study. The addition of cetuximab showed a statistically significant improvement in survival from 7.4 to 10.1 months $(P=$ 0.036). In a subset analysis, there was a greater benefit for the following subgroups: under 65 years of age, better performance status and the receipt of cisplatin as opposed to carboplatin. These data established the role of cetuximab in first-line therapy for advanced HNSCC when given in combination with cisplatin and 5-FU.

Cetuximab has also been shown to be active in combination with paclitaxel in the first-line setting. In a phase II study from the Spanish Head and Neck Cancer Group, 46 patients received first-line treatment with paclitaxel given once weekly at $80 \mathrm{mg} / \mathrm{m}^{2}$ plus cetuximab. ${ }^{72}$ Among the 35 evaluable patients, the combined $\mathrm{CR}$ and $\mathrm{PR}$ rate was $71 \%$ with a disease control rate of $88 \%$. Median PFS was 5 months. At a median follow-up of 5.6 months, the median overall survival had not been reached. This study suggests that cetuximab has additive anti-tumor activity with other cytotoxic agents. 


\section{Cetuximab in disease progressing on prior therapy}

The anti-tumor activity of cetuximab in patients with advanced head and neck cancer was apparent early on in phase 1 investigations. ${ }^{73,74}$ Cisplatin and cetuximab were combined in a single-institution phase $1 \mathrm{~b}$ study of 12 patients, 6 of whom had received prior systemic therapy after disease recurrence. ${ }^{75}$ In this dose-ranging study, cetuximab was administered at 3 different dose levels. A high percentage of EGFR binding in tumor tissue was achieved with an initial $400 \mathrm{mg} / \mathrm{m}^{2}$ loading dose and subsequent weekly doses of $250 \mathrm{mg} / \mathrm{m}^{2}$. Over all dose levels, 2 CRs and 4 PRs were noted among 9 evaluable patients (response rate, 67\%).

A major unmet medical need is the treatment of patients with incurable HNSCC who failed to respond to platinumbased chemotherapy. Several published studies have focused on this unfortunate patient population. In a European multicenter phase II trial performed by Baselga et al, 96 patients with platinum-refractory disease were treated by adding cetuximab to the platinum dose and schedule that the patients had previously failed. ${ }^{76}$ The response rate was $10 \%$, with a disease control rate of $53 \%$, median time to progression of 2.79 months and OS of 6.01 months. In a similar US-based phase II study performed by Herbst et al, 130 patients with stable disease (SD) or progressive disease (PD) on previous platinum therapy, received treatment with cetuximab and cisplatin. ${ }^{77}$ There were 2 PD cohorts: PD1 $(n=25)$ that had patients whose disease progressed on two cycles of protocolspecified platinum-based therapy and PD2 $(n=54)$ that had patients whose disease progressed within three months of any platinum-based therapy. The response rates were $18 \%$ for the SD cohort, 20\% for the PD1 cohort and 6\% for the PD2 cohort with median survivals of 11.7 months, 6.1 months and 4.3 months, respectively. Other than increased exposure to cisplatin in the PD2 cohort, there is little other information about biomarkers (such as EGFR expression) to explain the striking difference (albeit not statistically significant) in efficacy between these cohorts. These studies made it clear that cetuximab was active in these patients. What was not clear was whether the observed anti-tumor activity resulted from the combination of cisplatin plus cetuximab or if cetuximab itself was responsible.

A landmark phase II study led by Vermorken et al enrolled 103 patients actively failing platinum-based therapies and treated them with cetuximab as a monotherapy. They reported a response rate of $12.6 \%$, disease control rate of $46 \%$ and median overall survival of 5.84 months ${ }^{78}$ Based on contemporary data, the expected response rate to secondline chemotherapy in this population is $2.5 \%{ }^{68}$ Patients progressing on cetuximab monotherapy in this trial were given the option to receive cetuximab and cisplatin; there were no responses among 53 patients who received such combined treatment after progressing on cetuximab.

The comparable response rates and median overall survival (5-6 months) in these three studies support the conclusion that cetuximab monotherapy is the preferred approach for platinum-refractory patients with incurable HNSCC. Furthermore, the data in aggregate suggest that chemotherapy in this setting probably adds little to the observed response and clinical benefit conferred by cetuximab monotherapy. Vermorken and colleagues conducted a pooled analysis of these three phase II trials. ${ }^{79}$ The analysis compared the outcome of these patients to those in a retrospective study who received a range of common second-line treatment approaches including best supportive care, palliative chemotherapy, radiotherapy and chemoradiotherapy. The median OS for patients in the retrospective study was 3.4 months, roughly half the median OS time in the three phase II trials investigating the role of cetuximab in patients with advanced HNSCC who had progressed on platinum-based therapy.

While the study discussed above combining paclitaxel and cetuximab allowed patients in the first-line treatment setting for recurrent/metastatic disease, investigators have also evaluated taxane plus cetuximab therapy in the secondline setting in platinum pre-treated patients. In a study by Knoedler and colleagues, 84 patients received cetuximab and

Table I Phase II studies of cetuximab in disease progressing on prior therapy

\begin{tabular}{|c|c|c|c|c|c|c|}
\hline Study & Treatment & Patients (n) & Response (\%) & $\begin{array}{l}\text { Disease control } \\
\text { rate }(\%)\end{array}$ & $\begin{array}{l}\text { Median PFS } \\
\text { (months) }\end{array}$ & $\begin{array}{l}\text { Median OS } \\
\text { (months) }\end{array}$ \\
\hline Baselga et $\mathrm{a}^{76}$ & Cetuximab/platinum & 96 & 10 & 53 & 2.79 & 6.01 \\
\hline Herbst et $\mathrm{al}^{77}$ & Cetuximab/platinum & 155 & 11.5 & NA & $\begin{array}{l}\text { PDI (3.0) } \\
\text { PD2 (2.0) } \\
\text { SD (4.9) }\end{array}$ & $\begin{array}{l}\text { PDI (6.I) } \\
\text { PD2 (4.3) } \\
\text { SD (II.7) }\end{array}$ \\
\hline Vermorken et $\mathrm{al}^{78}$ & Cetuximab monotherapy & 103 & 13 & 46 & 2.29 & 5.84 \\
\hline
\end{tabular}

Abbreviations: PFS, progression-free survival; OS, overall survival; NA, not applicable; PDI, patients who progressed on prior platinum therapy on protocol; PD2, patients who had prior platinum therapy off study; SD, stable disease. 
docetaxel ( $35 \mathrm{mg} / \mathrm{m}^{2}$ on days $1,8,15$ of a 4 -week cycle) with a PR rate of $12 \%$, PFS of 4 months and OS of 7 months. ${ }^{80}$ While these numbers are modest, they do indicate some activity in a population of patients that tends to be highly refractory to therapy. In this phase 2 trial, however, the response rate and median survival results are similar to data observed in the cetuximab monotherapy studies.

It is reasonable to ask if the addition of other molecularly targeted therapies to cetuximab could achieve further improvements in outcome in platinum-refractory patients. In addition, nearly all responding patients will eventually become resistant to cetuximab, and thus future research is required to study ways to bypass resistance mechanisms. Preclinical models have suggested that vascular endothelial growth factor (VEGF) production by tumor cells may counteract the antitumor effects of EGFR inhibition. Thus, there is rationale for combined targeting in order to enhance anti-tumor activity. There is an ongoing phase II trial by Kies et al for patients with recurrent or metastatic HNSCC who have been previously treated with up to 1 chemotherapy regimen. ${ }^{81}$ Patients in this study receive weekly cetuximab along with bevacizumab every 3 weeks. Of 15 evaluable patients at the last interim analysis, 4 had a partial response and 8 had stable disease. Grade $3 / 4$ adverse events included proteinuria, hypertension, stomatitis, rash and fatigue. Results of this trial should provide additional insights, especially the planned biomarker analysis related to EGFR and angiogenesis.

\section{Tolerability}

Cetuximab may cause significant grade 4 infusion reactions in a minority $(\sim 3 \%)$ of patients. Interestingly, centers in southeastern US note a significantly higher incidence of infusion reactions. A recent study showed that $22 \%$ of patients treated with cetuximab in 3 centers from Tennessee and North Carolina had severe hypersensitivity reactions. ${ }^{82}$ A history of prior allergy or atopy was identified as a risk factor. A study by Chung et al showed that in 17 of 21 patients who had experienced such an allergic reaction there were preexisting IgE antibodies against galactose- $\alpha-1,3$-galactose, an oligosaccharide present on cetuximab. ${ }^{83}$ It is unclear why people in one area of the country have a strikingly higher prevalence of these IgE antibodies against galactose- $\alpha-1,3-$ galactose, whereas in other areas the incidence of such $\operatorname{IgE}$ antibodies is very low. Whether one could test for the presence of such antibodies before administering cetuximab and thereby avoid grade 4 infusion reactions is the subject of ongoing research.
The most common toxicity of cetuximab occurs in the skin with a characteristic acne-like pustular rash often accompanied by xerosis, nail changes, paronychia and digital fissuring. This is seen in $>80 \%$ of patients treated with cetuximab. EGFR is expressed at the basal layer of the epidermis and it is, therefore, not surprising that the skin expresses this mechanism-based toxicity. ${ }^{84}$ The rash is usually managed with emollients, topical glucocorticoids, and oral antibiotics such as doxycycline and minocycline.

There have also been multiple case-reports of cetuximabrelated ocular toxicities including corneal erosions, keratitis and trichomegaly of eyelashes. ${ }^{85-87}$ These effects are not surprising given the role of EGFR family members in the cornea healing process. ${ }^{88,89}$

The presence of rash as a positive predictor of improved outcome with EGFR inhibitors including cetuximab was suggested initially in studies of cetuximab in HNSCC and colorectal cancer. In the phase 2 trial conducted by Herbst et al, for example, patients without rash had a response rate of $0 \%$ and 1 -year survival of $18 \%$ compared to a $25 \%$ response rate and 1-year survival of $55 \%$ in those with grade 2 or 3 rash. ${ }^{77}$

Despite the skin toxicities caused by cetuximab, the risk of poor wound healing appears to be low. In a subset analysis of 39 patients treated with radiation or radiation plus cetuximab who then proceeded to have neck dissections (6-8 weeks after treatment), there was no significant differences in time to healing or complication rates between the two groups..$^{90}$ A retrospective case series of 35 patients who underwent salvage neck dissections after treatment with radiation alone or with cetuximab also showed no significant increase in risk for post-surgical wound complications -2 of 15 patients treated with radiation and cetuximab had wound healing complications while no complications occurred in the 20 patients who received radiation alone $(P=0.20) .{ }^{91}$ It will be important to continue collecting data on patients undergoing major surgery who have received EGFR inhibitor therapy.

Hypomagnesemia is another class effect associated with anti-EGFR antibodies, including cetuximab. This appears to be due to inhibition of magnesium reabsorption in the ascending loop of Henle, which is a direct consequence of EGFR blockade in the kidney. Routine monitoring and repletion of potassium and magnesium is therefore necessary in patients receiving cetuximab. The onset of hypomagnesemia starts shortly after initiation of cetuximab and resolves within weeks of discontinuation. However, in patients with metastatic disease who require long-term treatment with 
Table 2 Studies of cetuximab in the first-line setting for recurrent/metastatic disease

\begin{tabular}{llllll}
\hline Study & Treatment & Patients $(\mathbf{n})$ & Response (\%) & Median PFS (months) & Median OS (months) \\
\hline Burtness et al & CDDP & 57 & 10 & 2.7 & 8 \\
& CDDP/cetuximab & 60 & $26(P=0.03)$ & $4.2(P=0.09)$ & $9.2(\mathrm{NS})$ \\
Hitt et al $^{72}$ & Cetuximab/paclitaxel & 46 & 71 & 5 & NA \\
Vermorken et al $^{71}$ & CDDP/FU & 220 & 20 & 3.3 & 7.4 \\
& CDDP/FU/cetuximab & 222 & $36(P<0.001)$ & $5.6(P<0.001)$ & $10.1(P=0.036)$ \\
\hline
\end{tabular}

Abbreviations: PFS, progression-free survival; OS, overall survival; CDDP, cisplatin; FU, fluorouracil; NA, not applicable.

cetuximab, ongoing magnesium supplementation may be necessary, including parenteral replacement.

Overall, cetuximab treatment has an acceptable tolerability profile, with the majority of adverse events being mild or moderate in severity and clinically manageable. Of note, in the clinical trials to date, it has been feasible to combine cetuximab with diverse cytotoxic chemotherapy regimens without significantly increasing the adverse effects commonly seen with such regimens when given without cetuximab. In the EXTREME trial, for example, there was no significant difference in the overall incidence of grade 3 or 4 adverse events between the groups except for skin reactions $(P<0.001)$, hypomagnesemia $(P=0.05)$, anorexia $(P=0.05)$ and sepsis $(P=0.02){ }^{71}$

\section{Ongoing and future studies with cetuximab in recurrent/ metastatic disease}

While cetuximab does result in improved survival in combination with chemotherapy and radiation, single agent response rates in the platinum-refractory setting are modest. Alternate (bypass) signaling pathways are undoubtedly responsible for resistance to cetuximab. Targets of interest include angiogenesis, the insulin-like growth factor receptor (IGF-1R), the phospho-inositol 3 kinase/Akt/mTOR axis, and Src. There are a number of available agents that antagonize these pathways and therefore might have utility in combination with cetuximab to overcome such resistance. In particular, inhibition of IGF-1R is actively being studied in head and neck cancer. Preclinical data from head and neck cell lines have indicated that IGR-1R is elevated compared to normal tissue. In addition, treatment of head and neck tumor xenografts with cetuximab and the IGF-1R monoclonal antibody IMC-A12 resulted in tumor regression. ${ }^{92}$ Clinical trials are underway to evaluate this combination in the clinic.

Activated Akt has been shown to be associated with a worse prognosis in HNSCC as well, and trials are under- way to study the safety and effectiveness of combining cetuximab and drugs inhibiting mTOR, a target downstream from Akt. ${ }^{93}$ Preclinical data have shown that the mTOR inhibitor rapamycin has anti-tumor activity in HNSCC in vivo. ${ }^{94}$ Thus, trials are ongoing to assess the activity of newer generation of mTOR inhibitors (temsirolimus, everolimus) with cisplatin and cetuximab in patients with metastatic disease.

The Src family of kinases has been implicated in the pathogenesis of head and neck cancer. ${ }^{95,96}$ In addition, Grandis and colleagues have shown that combined inhibition of the Src and EGFR kinases inhibits HNSCC cell growth and invasion. ${ }^{97}$ Currently, there is an ongoing phase I study to test the safety of cetuximab and the Src inhibitor dasatinib in patients with advanced malignancies, which will provide more clinical data with regards to the safety of this combination.

Table 3 lists selected studies that are ongoing to assess the safety and effectiveness of cetuximab in combination with novel targeted agents. As these studies evolve and move into efficacy studies in selected diseases including HNSCC, we will learn whether combining targeted agents with cetuximab will have more of an impact on the treatment of recurrent disease and the mitigation of resistance to cetuximab-based therapies.

\section{Biomarkers of response}

While cetuximab is a promising treatment for HNSCC, response rates to cetuximab monotherapy are low, and clearly not all patients benefit from this drug. There is a great deal of interest in the field to determine biomarkers that could predict response to therapy. In E5397, analysis of EGFR expression levels was performed and the results correlated with patient outcomes. ${ }^{69}$ Tumors were classified as very high EGFR immunoreactive if $3+$ staining was present on $>80 \%$ of cells and low-moderate if there was a lesser degree of staining. The treatment groups were well-balanced with regard to the EGFR immunoreactivity 
Table 3 Ongoing studies combining cetuximab with novel agents for recurrent/metastatic disease (available on clinicaltrials.gov)

\begin{tabular}{|c|c|c|c|c|}
\hline Title & Treatment & Phase & Sample size & Study drug target \\
\hline $\begin{array}{l}\text { A phase II trial of cetuximab and } \\
\text { bevacizumab in patients with recurrent } \\
\text { or metastatic head and neck cancer } \\
\text { NCT00409565 }\end{array}$ & $\begin{array}{l}\text { cetuximab } 400 \mathrm{mg} / \mathrm{m}^{2} \text { loading } \\
\text { followed by } 250 \mathrm{mg} / \mathrm{m}^{2} \text { weekly, } \\
\text { bevacizumab } 15 \mathrm{mg} / \mathrm{kg} \text { q2I days }\end{array}$ & II & 48 & VEGF \\
\hline $\begin{array}{l}\text { Cetuximab with or without sorafenib } \\
\text { tosylate in treating patients with refractory, } \\
\text { recurrent and/or metastatic head and } \\
\text { neck cancer NСT00939627 }\end{array}$ & $\begin{array}{l}\text { cetuximab weekly, placebo versus } \\
\text { cetuximab weekly, sorafenib twice } \\
\text { daily days I-2I }\end{array}$ & II & 88 & $\begin{array}{l}\text { Raf; VEGFR I, 2, 3; } \\
\text { Flt-3; PDGFR } \beta \text {; } \\
\text { c-kit, RET }\end{array}$ \\
\hline $\begin{array}{l}\text { Cilengitide in recurrent and/or } \\
\text { metastatic squamous cell carcinoma } \\
\text { of the head and neck NCT007050I6 }\end{array}$ & $\begin{array}{l}\text { Cilengitide, cetuximab, cisplatin, } \\
5 \mathrm{FU} \text { vs cetuximab, cisplatin, 5FU }\end{array}$ & $1 / I I$ & 195 & Integrins \\
\hline $\begin{array}{l}\text { Study of IMC-A I 2, alone or in combination } \\
\text { with cetuximab, in patients with recurrent } \\
\text { or metastatic squamous cell carcinoma } \\
\text { of the head and neck NCT006I7734 }\end{array}$ & IMC-AI $2 \pm$ cetuximab & II & 90 & IGF-IR \\
\hline $\begin{array}{l}\text { BIBW } 2992 \text { in head and neck cancer } \\
\text { NCT005 I } 4943\end{array}$ & cetuximab vs BIBW2992 & II & 100 & EGFR, Her-2/neu \\
\hline Phase I study of cetuximab and RAD00 I & cetuximab and RAD00I & I & & mTOR \\
\hline $\begin{array}{l}\text { Cisplatin, cetuximab and temsirolimus } \\
\text { in HNSCC NCTOI0I5664 }\end{array}$ & cisplatin, cetuximab, temsirolimus & $\mathrm{I} / \mathrm{II}$ & 56 & mTOR \\
\hline $\begin{array}{l}\text { RAD00I in combination with cetuximab } \\
\text { and cisplatin in recurrent/metastatic } \\
\text { HNSCC NCT01009346 }\end{array}$ & cisplatin, cetuximab, RAD00 I & $1 / I I$ & 98 & mTOR \\
\hline $\begin{array}{l}\text { Safety study of cetuximab plus } \\
\text { dasatinib (BMS-354825) NCT00388427 }\end{array}$ & cetuximab and dasatinib & I & 30 & Src \\
\hline
\end{tabular}

scores, with about a third of the patients in each group having very high immunoreactivity. The addition of cetuximab significantly increased response rate in the moderate staining group, with no apparent benefit from the addition of cetuximab for the high immunoreactive group. Thus, it was hypothesized that EGFR expression could be a marker for cetuximab activity, with high EGFR expressing tumors being more resistant to cetuximab, perhaps due to the inability to fully saturate a greater number of receptors with the drug. This observation has not been confirmed, however, in subsequent studies, including the EXTREME trial, in which the high EGFR expressors did derive a survival benefit from cetuximab-containing therapy. In addition, tissue from the phase II trial by Herbst and colleagues discussed above was analyzed using the EGFR staining intensity and density scoring system, and also showed that EGFR expression levels did not predict for response to cetuximab. ${ }^{98}$ Thus, this methodology does not appear to be a useful approach to guide therapy. It is not known if newer automated, quantitative immunohistochemical techniques, which allow for more precise quantification of EGFR levels as well as subcellular localization will have more power to predict for cetuximab response.
EGFR gene copy number detected by FISH (fluorescence in situ hybridization) has been analyzed as well. In one retrospective study, $63 \%$ of HNSCC tumors had high EGFR gene copy numbers, which were associated with a trend towards an inferior recurrence-free survival. ${ }^{99}$ However, when tumors from the EXTREME trial were analyzed for EGFR gene copy number by FISH, being FISH+ with this methodology was not a predictive marker for cetuximab efficacy. ${ }^{100}$ Thus, increased EGFR copy number was not associated with an improvement in overall survival in either treatment arm or a response to cetuximab.

Finally, there are more recent data regarding the potential utility of matrix-assisted laser desorption ionization (MALDI) mass spectrometry (MS) to predict which patients may benefit from EGFR inhibitors. Utilizing MALDI-MS to analyze serum, Chung and colleagues have identified a favorable proteomic profile that may predict for response to cetuximab. ${ }^{101}$ In a retrospective analysis of 314 patient samples, the favorable profile predicted for a survival benefit in cohorts of patients that were treated with EGFR inhibitors. This proteomic profile did not predict for an increase in survival in a control group of patients that were not treated with EGFR inhibitors. This novel technique will need to be studied more in future studies. 


\section{Conclusions and future directions for the treatment of advanced disease}

Cetuximab is now firmly established as an active treatment either alone or in combination with radiation or chemotherapy for the treatment of advanced recurrent/metastatic HNSCC. It is clear that in the first-line treatment setting for recurrent/metastatic disease, the addition of cetuximab to a platinum doublet significantly improves overall median survival of patients with incurable HNSCC. For those patients who have not received cetuximab previously for recurrent/metastatic disease and who are platinum refractory, cetuximab monotherapy has emerged as the standard of care with response rates that are superior to those seen with any second-line chemotherapy drug in this setting. ${ }^{78}$ The limited data available with cetuximab and taxanes suggest that this may be an active combination as well, especially for patients who cannot receive platinum drugs. There are currently no randomized clinical trials data comparing a taxane/cetuximab regimen to cetuximab alone in any setting, and therefore we do not know the true magnitude of the contribution of cetuximab to the activity of the regimen. Given the paucity of treatment options for this patient population, participation in clinical trials assessing novel combinations that incorporate cetuximab remains a very reasonable approach.

Determining biomarkers that could predict for a response and survival benefit with cetuximab will be a key focus of future studies. We also do not understand the determinants of de novo or acquired resistance to cetuximab. We suspect that tumors with an EGFR vIII variant and therefore lacking the cetuximab-binding domain will be resistant, but this has not been formally shown. In addition, as patients are exposed to EGFR inhibitors earlier in the treatment course and in some cases as part of their initial treatment with radiation for curative intent, the utility of re-treatment with cetuximab for recurrent disease is unknown.

A major focus of ongoing and future research is to study the safety and efficacy of cetuximab in combination with other EGFR inhibitors as well as other targeted therapies. Currently, trials are underway evaluating simultaneous targeting of multiple signaling pathways such as EGFR, insulin-like growth factor receptor, Akt and Src. For patients who have a hypersensitivity reaction to cetuximab, panitumumab may be a reasonable alternative although data from studies testing the activity of panitumumab in HNSCC are still quite limited.
The conventional loading dose, followed by a weekly maintenance schedule of cetuximab has been used in all of the clinical trials leading to its approval. This schedule can be inconvenient for patients though, as it requires weekly infusion room visits. There is an ongoing trial to study cetuximab dosing on an every-other-week schedule. ${ }^{102}$ If this proves to be feasible and efficacious, it may support future research to validate the every-other-week schedule as therapeutically equivalent to the conventional weekly schedule.

Finally, HNSCC that is related to the human papillomavirus (HPV) is emerging as a different disease compared to the traditional smoking- and alcohol-related HNSCC. HPV-associated HNSCC has been shown to have a generally favorable prognosis, and these tumors tend to be chemotherapy and radiotherapy sensitive. ${ }^{6}$ In a small study utilizing induction therapy for locally advanced HNSCC, tumors that were HPV, p16+ had an increased response to platinum and 5-FU. ${ }^{103}$ Biomarker analysis of the patient samples also indicated that HPV status and EGFR expression by immunohistochemistry were inversely related, with HPV+, low EGFR expressor having the best response to treatment and prognosis. ${ }^{104}$ Patients in this study did not receive cetuximab as part of their induction regimen, and therefore it is not known how the favorable prognosis group would respond to EGFR inhibition. Given the data, although from a small series, that HPV+ tumors have lower EGFR expression, one important question is whether cetuximab will improve the outcome of HPV+ disease in combination with chemotherapy. An even more provocative idea is whether cetuximab could be used in place of cytotoxic therapy. Analysis of specimens from existing studies will likely provide more insight into the biology of HPV+ HNSCC. In the future, trials will probably study these two subtypes of HNSCC separately, and in that context it will be useful to determine how effective cetuximab is for the treatment of HPV+, p16+ HNSCC. ${ }^{105}$

\section{Disclosures}

$\mathrm{RBC}$ is a member of the BMS/ImClone Speakers' Bureau. MT and RM declare no conflicts of interest.

\section{References}

1. Jemal A, Siegel R, Ward E, Murray T, Xu J, Thun MJ. Cancer Statistics. CA Cancer J Clin. 2007;57:43-66.

2. Goldenberg D, Lee J, Koch WM, et al. Habitual risk factors for head and neck cancer. Otolaryngal Head Neck Surg. 2004;131:986-993.

3. Herrero R, Castellsague X, Pawlita M. Human papillomavirus and oral cancer: the International Agency for Research on Cancer multicenter study. J Natl Cancer Inst. 2003;95:1772-1183.

4. D'Souza G, Agrawal Y, Halpern J, Bodison S, Gillison ML. Oral sexual behaviors associated with prevalent oral human papillomavirus infection. J Infect Dis. 2009;199:1263-129. 
5. D'Souza G, Kreimer AR, Viscidi R, et al. Case-control study of human papillomavirus and oropharyngeal cancer. $N$ Engl J Med. 2007;356:1944-1956.

6. Fakhry C, Westra WH, Li S, et al. Improved survival of patients with human papillomavirus-positive head and neck squamous cell carcinoma in a prospective clinical trial. J Natl Cancer Inst. 2008;100:261-269.

7. Bonner JA, Harari PM, Giralt J, et al. Radiotherapy plus cetuximab for squamous-cell carcinoma of the head and neck. $N$ Engl $J$ Med. 2006;354:567-578.

8. Astsaturov I, Cohen RB, Harari PM. EGFR-targeting monoclonal antibodies in head and neck cancer. Curr Cancer Drug Targets. 2006;6:691-710

9. Mehra R, Cohen RB, Burtness BA. The role of cetuximab for the treatment of squamous cell carcinoma of the head and neck. Clin $A d v$ Hematol Oncol. 2008;6:742-750.

10. Panikkar RP, Astsaturov I, Langer CJ. The emerging role of cetuximab in head and neck cancer: a 2007 perspective. Cancer Invest. 2008;26:96-103

11. Messa C, Russo F, Caruso MG, Di Leo A. EGF, TGF-alpha, and EGF-R in human colorectal adenocarcinoma. Acta Oncol. 1998;37:285-289.

12. Lax I, Fischer R, $\mathrm{Ng} \mathrm{C}$, et al. Noncontiguous regions in the extracellular domain of EGF receptor define ligand-binding specificity. Cell Regul. 1991;2:337-345.

13. Prigent SA, Lemoine NR. The type 1 (EGFR-related) family of growth factor receptors and their ligands. Prog Growth Factor Res. 1992;4:1-24.

14. Oc P, Rhys-Evans PH, Modjtahedi H, Eccles SA. The role of c-erbB receptors and ligands in head and neck squamous cell carcinoma. Oral Oncol. 2002;38:627-640.

15. Hambek M, Baghi M, Baumaun H. ZD 1839 inhibits phosphorylation of three different downstream signal transducers in head and neck cancer. Anticancer Res. 2005;25:1871-1875.

16. Haura E, Zheng Z, Song L, Cantor A, Bepler G. Activated epidermal growth receptor-Stat3 signaling promotes tumor survival in vivo in non-small cell lung cancer. Clin Cancer Res. 2005;11:8288-8294.

17. Lo H, Hsu S, Ali-Sayed M. Nuclear interaction of EGFR and STAT3 in the activation of the iNOS/NO pathway. Cancer Cell. 2005;7:575-589.

18. Wanner G, Mayer C, Kehlbach R, Rodemann H, Dittmann K. Activation of protein kinase $\mathrm{C}$ epsilon stimulates DNA repair via epidermal growth factor nuclear accumulation. Radiother Oncol. 2008;86:383-390.

19. Chen D, Nirodi C. The epidermal growth factor receptor: a role in repair of radiation-induced DNA damage. Clin Cancer Res. 2007;13:6555-6560.

20. Rischin D, Peters L, Hicks R. Phase I trial of concurrent tirapazamine, cisplatin and radiotherapy in patients with advanced head and neck cancer. J Clin Oncol. 2001;19:535-542.

21. Friedmann B, Caplin M, Hartley J, Hochhauser D. Modulation of DNA repair in vitro after treatment with chemotherapeutic agents by the epidermal growth factor receptor inhibitor ZD1839. Clin Cancer Res. 2004;10:6476-6486

22. Yacoub A, McKinstry R, Hinman D, Chung T, Dent P, Hagan M. Epidermal growth factor and ionizing radiation up-regulate the DNA repair genes XRCC1 and ERCC1 in DU145 and LNCaP prostate carcinoma through MAPK signaling. Radiat Res. 2003;159:439-4352.

23. Huang S, Harari P. Modulation of radiation response after epidermal growth factor receptor blockade in squamous cell carcinomas: inhibition of damage repair, cell cycle kinetics and tumor angiogenesis. Clin Cancer Res. 2000;6:2166-2174.

24. Ang KK, Berkey BA, Tu X, et al. Impact of epidermal growth factor receptor expression on survival and pattern of relapse in patients with advanced head and neck carcinoma. Cancer Res. 2002;62:7350-7256.

25. Sheridan MT, O’Dwyer T, Seymour CB, Mothersill CE. Potential indicators of radiosensitivity in squamous cell carcinoma of the head and neck. Radiat Oncol Investig. 1997;5:180-186.
26. Dassonville O, Formento JL, Francoual M, et al. Expression of epidermal growth factor receptor and survival in upper aerodigestive tract cancer. J Clin Oncol. 1993;11:1873-1878.

27. Rubin JG, Melhem MF, Gooding WE, et al. Levels of TGF-alpha and EGFR protein in head and neck squamous cell carcinoma and patient survival. J Natl Cancer Inst. 1988;998:824-832.

28. Lynch TJ, Bell DW, Sordella R, et al. Activating mutations in the epidermal growth factor receptor underlying responsiveness of non-small-cell lung cancer to gefitinib. $N$ Engl J Med. 2004;350:2129-2139.

29. Pao W, Miller V, Zakowski M, et al. EGF receptor gene mutations are common in lung cancers from "never smokers" and are associated with sensitivity of tumors to gefitinib and erlotinib. Proc Natl Acad Sci U SA. 2004;101:13306-13311.

30. Loeffler-Ragg J, Witsch-Baumgartner M, Tzankov A, et al. Low incidence of mutations in EGFR kinase domain in Caucasian patients with head and neck squamous cell carcinoma. Eur J Cancer. 2006;42:109-111.

31. Sheikh Ali MA, Gunduz M, Nagatsuka H, et al. Expression and mutation analysis of epidermal growth factor receptor in head and neck squamous cell carcinoma. Cancer Sci. 2008;99:1589-1594.

32. Sok J, Coppelli F, Thomas S. Mutant epidermal growth factor receptor (EGFRvIII) contributes to head and neck cancer growth and resistance to EGFR targeting. Clin Cancer Res. 2006;12:5064-5073.

33. Harari P, Huang S. Head and neck cancer as a clinical model for molecular targeting of therapy: combining EGFR blockade with radiation. Int J Radiat Oncol Biol Phys. 2001;49:427-433.

34. Huang S, Bock J, Harari P. Epidermal growth factor receptor blockade with $\mathrm{C} 225$ modulates proliferation, apoptosis and radiosensitivity in squamous cell carcinomas of the head and neck. Cancer Res. 1999;59:1935-1940.

35. Feng F, Lopez C, Normolle D. Effect of epidermal growth factor receptor inhibitor class in the treatment of head and neck cancer with concurrent radiochemotherapy in vivo. Clin Cancer Res. 2007; 13:2512-2518.

36. Fan Z. Antitumor effect of anti-epidermal growth factor receptor monoclonal antibodies plus cis-diamminedichloroplatinum on well established A431 cell xenografts. Cancer Res. 1993;53: 4637-4642.

37. Brown D. Antiepidermal growth factor receptor antibodies augment cytotoxicity of chemotherapeutic agents on squamous cell carcinoma cell lines. Otolaryngal Head Neck Surg. 2000;122:75-83.

38. Goldstein NI, Prewett M, Zuklys K, Rockwell P, Mendelsohn J. Biological efficacy of a chimeric antibody to the epidermal growth factor receptor ina human tumor xenograft model. Clin Cancer Res. 1995;1:1311-1318.

39. Baselga J. The EGFR as a target for anticancer therapy-focus on cetuximab. Eur J Cancer. 2001;37:S16-S22.

40. Ciardiello F, Tortora G. A novel approach in the treatment of cancer: targeting the epidermal growth factor receptor. Clin Cancer Res. 2001;7:2958-29570.

41. Li S, Schmitz K, Jeffrey P, Wiltzius J, Kussie P, Ferguson F. Structural basis for inhibition of the epidermal growth factor receptor by cetuximab. Cancer Cell. 2005;7:301-311.

42. Sigismund S, Woelk T, Puri C. Clathrin-independent endocytosis of ubiquitinated cargos. Proc Natl Acad Sci U S A. 2005;102:2760-2765.

43. Lopez-Albaitero A, Ferris R. Immune activation by epidermal growth factor receptor specific monoclonal antibody therapy for head and neck cancer. Arch Otolaryngol Head Neck Surg. 2007;133:1277-1281.

44. Kurai J, Chikumi H, Hashimoto K. Antibody-dependent cellular cytotoxicity mediated by cetuximab against lung cancer cell lines. Clin Cancer Res. 2007;13:1552-1561.

45. Lopez-Albaitero A, Lee SC, Morgan S, et al. Role of polymorphic Fc gamma receptor IIIa and EGFR expression level in cetuximab mediated, NK cell dependent in vitro cytotoxicity of head and neck squamous cell carcinoma cells. Cancer Immunol Immunother. 2009;58:1855-1864. 
46. Wirth LJ PM, Tishler RB, Haddad RI, Goguen L, Clark JR, Allen AM. Phase I study of panitumumab, chemotherapy and intensity-modulated radiotherapy (IMRT) for head and neck cancer (HNC): Early results. J Clin Oncol. 2008;26(May 20 suppl):abstr 6007.

47. Kuenen B, Witteveen E, Ruijter R, et al. A phase I study of IMC-11F8, a fully human anti-epidermal growth factor receptor (EGFR) IgG1 monoclonal antibody in patients with solid tumors. Interim results. J Clin Oncol. 2006;24(18S, June 20 suppl):abstr 3024.

48. Johns TG, Adams TE, Cochran JR, et al. Identification of the epitope for the epidermal growth factor receptor-specific monoclonal antibody 806 reveals that it preferentially recognizes an untethered form of the receptor. J Biol Chem. 2004;279:30375-30384.

49. Li S, Schmitz KR, Jeffrey PD, Wiltzius JJ, Kussie P, Ferguson KM. Structural basis for inhibition of the epidermal growth factor receptor by cetuximab. Cancer Cell. 2005;7:301-311.

50. Talavera A, Friemann R, Gomez-Puerta S, et al. Nimotuzumab, an antitumor antibody that targets the epidermal growth factor receptor, blocks ligand binding while permitting the active receptor conformation. Cancer Res. 2009;69:5851-5859.

51. Snyder LC, Astsaturov I, Weiner LM. Overview of monoclonal antibodies and small molecules targeting the epidermal growth factor receptor pathway in colorectal cancer. Clin Colorectal Cancer. 2005;5 Suppl 2: S71-S80.

52. Schmiedel J, Blaukat A, Li S, Knochel T, Ferguson KM. Matuzumab binding to EGFR prevents the conformational rearrangement required for dimerization. Cancer Cell. 2008;13:365-373.

53. Pfister DG, Su YB, Kraus DH, et al. Concurrent cetuximab, cisplatin, and concomitant boost radiotherapy for locoregionally advanced, squamous cell head and neck cancer: a pilot phase II study of a new combined-modality paradigm. J Clin Oncol. 2006;24:1072-1078.

54. Langer CJ, Li JW, Patel UA, et al. Preliminary analysis of ECOG 3303: Concurrent radiation (RT), cisplatin (DDP) and cetuximab (C) in unresectable, locally advanced (LA) squamous cell carcinoma of the head and neck (SCCHN). J Clin Oncol. 2008;26(May 20 suppl):abstr 6006.

55. Koutcher L, Fury M, Wolden S, et al. Comparison of cisplatin and radiation to cetuximab and RT for locally advanced head and neck cancer: a preliminary analysis. J Clin Oncol. 2009;27(15s):abstr 6042.

56. Argiris AE, Gibson MK, Heron DE, et al. Phase II trial of neoadjuvant docetaxel $(\mathrm{T})$, cisplatin $(\mathrm{P})$, and cetuximab $(\mathrm{E})$ followed by concurrent radiation, $\mathrm{P}$ and $\mathrm{E}$ in locally advanced head and neck cancer. J Clin Oncol. 2007; Pt I. Vol 25, No. 18S (June 20 suppl):abstr 6051.

57. Wanebo H, Ghebremichael M, Burtness B, Spencer S, Ridge J, Forastiere A. Phase II evaluation of cetuximab (C225) combined with induction paclitaxel and carboplatin followed by C225, paclitaxel, carboplatin, and radiation for stage III/IV operable squamous cancer of the head and neck (ECOG, E2303). J Clin Oncol. 2007; Pt I, Vol 25, No. 18S (June 20 Supplement):abstr 6015.

58. Kies MS, Holsinger FC, Lee JJ, et al. Induction chemotherapy and cetuximab for locally advanced squamous cell carcinoma of the head and neck: results from a phase II prospective trial. J Clin Oncol. 2010;28:8-14.

59. Vermorken JB, Remenar E, van Herpen C, et al. Cisplatin, fluorouracil, and docetaxel in unresectable head and neck cancer. $N$ Engl J Med. 2007;357:1695-1704.

60. Posner MR, Hershock DM, Blajman CR, et al. Cisplatin and fluorouracil alone or with docetaxel in head and neck cancer. $N$ Engl J Med. 2007;357:1705-1715.

61. Tishler RB, Posner MR, Wirth LJ, et al. Cetuximab added to docetaxel, cisplatin, 5-fluorouracil induction chemotherapy in patients with newly diagnosed locally advanced head and neck cancer: a phase I study. J Clin Oncol. 2008;26(May 20 suppl):abstr 6001.

62. Mesia R, Vazquez S, Grau JJ, et al. A single-arm phase II trial to evaluate the combination of cetuximab plus docetaxel, cisplatin and 5-fluorouracil (TPF) as induction chemotherapy in patients with unresectable SCCHN. J Clin Oncol. 2009;27:15S(suppl):abstr 6015.
63. Richey LM, Shores CG, George J, et al. The effectiveness of salvage surgery after the failure of primary concomitant chemoradiation in head and neck cancer. Otolaryngol Head Neck Surg. 2007;136:98-103.

64. Mendenhall WM, Mendenhall CM, Malyapa RS, Palta JR, Mendenhall NP. Re-irradiation of Head and Neck Carcinoma. Am J Clin Oncol. 2008;31:393-398.

65. Tannock IF. Chemotherapy for head and neck cancer. J Otolaryngol. 1984;13:99-104.

66. Al-Sarraf M. Chemotherapy strategies in squamous cell carcinoma of the head and neck. Crit Rev Oncol Hematol. 1984;1:323-355.

67. Glick JH, Zehngebot LM, Taylor SGT. Chemotherapy for squamous cell carcinoma of the head and neck: A progress report. Am J Otolaryngol. 1980;1:306-323.

68. Leon X, Hitt R, Constenla M, et al. A retrospective analysis of the outcome of patients with recurrent and/or metastatic squamous cell carcinoma of the head and neck refractory to a platinum-based chemotherapy. Clin Oncol. 2005;17:418-424.

69. Burtness B, Goldwasser MA, Flood W; Phase II randomized trial of cisplatin plus placebo compared with cisplatin plus cetuximab in metastatic/recurrent head and neck cancer: an Eastern Cooperative Oncology Group study. J Clin Oncol. 2005;23:8646-88654.

70. Bourhis J, Rivera F, Mesia R, et al. Phase I/II study of cetuximab in combination with cisplatin or carboplatin and fluorouracil in patients with recurrent or metastatic squamous cell carcinoma of the head and neck. J Clin Oncol. 2006;24:2866-2872.

71. Vermorken JB, Mesia R, Rivera F. Platinum based chemotherapy plus cetuximab in head and neck cancer. $N$ Engl J Med. 2008;359: $1116-1127$

72. Hitt R, Irigoyen A, Nunez J. Phase II study of combination cetuximab and weekly paclitaxel in patients with metastatic/recurrent squamous cell carcinoma of head and neck: Spanish Head and Neck Cancer Group (TTCC). J Clin Oncol. 2007;P I. Vol 25, No. 18S(June 20 suppl):abstr 6012.

73. Baselga J, Pfister D, Cooper M, et al. Phase I studies of anti-epidermal growth factor receptor chimeric monoclonal antibody C225 alone and in combination with cisplatin. J Clin Oncol. 2000;18:314-904.

74. Robert F, Ezekiel M, Spencer S. Phase I study of anti-epidermal growth factor receptor antibody cetuximab in combination with radiation therapy in patients with advanced head and neck cancer. J Clin Oncol. 2001;19:3234-3243.

75. Shin DM, Donato NJ, Perez-Soler R, et al. Epidermal growth factor receptor targeted therapy with $\mathrm{C} 225$ and cisplatin in patients with head and neck cancer. Clin Cancer Res. 2001;7:1204-1213.

76. Baselga J, Trigo JM, Bourhis J. Phase II mulitcenter study of the antiepidermal growth factor receptor monoclonal antibody cetuximab in combination with platinum-based chemotherapy in patients with platinum-refractory metastatic and/or recurrent squamous cell carcinoma of the head and neck. $J$ Clin Oncol. 2005;23:5568-5577.

77. Herbst RS, Arquette M, Shin DM. Phase II multicenter study of the epidermal growth factor receptor antibody cetuximab and cisplatin for recurrent and refractory squamous cell carcinoma of the head and neck. J Clin Oncol. 2005;23:5568-5577.

78. Vermorken JB, Trigo J, Hitt R. Open-label, uncontrolled, multicenter phase II study to evaluate the efficacy and toxicity of cetuximab as a single agent in patients with recurrent and/or metastatic squamous cell carcinoma of the head and neck who failed to respond to platinum-based therapy. J Clin Oncol. 2007;25:2171-2177.

79. Vermorken J, Herbst R, Leon X, Amellal N, Baselga J. Overview of the efficacy of cetuximab in recurrent and/or metastatic squamous cell carcinoma of the head and neck in patients who previously failed platinum-based therapies. Cancer. 2008;112:2710-2719.

80. Knoedler MK, Gauler T, Matzdorff A, et al. Multicenter phase II study of cetuximab plus docetaxel in 84 patients with recurrent or metastatic, platinum-pretreated SCCHN. J Clin Oncol. 2009;27:15S(supp1):abstr 6048 . 
81. Kies M, Gibson M, Kim S, et al. Cetuximab and bevacizumab in patients with recurrent or metastatic head and neck squamous cell carcinoma: an interim analysis. J Clin Oncol. 2008;26(May 20 suppl):abstr 6072.

82. O'Neil B, Allen R, Spigel D. High incidence of cetuximab-related infusion reactions in Tennessee and North Carolina and the association with atopic history. J Clin Oncol. 2007;25:3644-3648.

83. Chung C, Mirakhur B, Chan E. Cetuximab-induced anaphylaxis and IgE specific for galactose-alpha-1,3-galactose. $N$ Engl J Med. 2008;358:1109-1117.

84. Yano S, Kondo K, Yamaguchi M. Distribution and function of EGR in human tissue and the effect of EGFR tyrosine kinase inhibition. Anticancer Res. 2003;23:3639-3650.

85. Foerster C, Cursiefen C, Kruse F. Persisting corneal erosion under cetuximab treatment: case report. Cornea. 2008;27:612-614.

86. Bouche O, Brixi-Benmansour H, Bertin A. Trichomegaly of the eyelashes following treatment with cetuximab. Ann Oncol. 2005;16:17111712.

87. Specenier P, Koppen C, Vermorken J. Diffuse punctate keratitis in a patient treated with cetuximab as monotherapy: letters to the editor. Ann Oncol. 2007;18:961-962.

88. Liu Z, Carvajal M, Carraway K. Expression of the receptor tyrosine kinases, epidermal growth factor receptor, ErbB2 and ErbB3 in human ocular surface epithelia. Cornea. 2001;20:81-85.

89. Nakamura Y, Sotozono C, Kinoshita S. The epidermal growth factor receptor: role in corneal wound healing and homeostasis. Exp Eye Res. 2001;72:511-517.

90. Harari P, Durland W, Chinnaiyan P, Hartig G. Impact of the EGFR inhibitor $\mathrm{C} 225$ on wound healing in advanced head and neck cancer patients undergoing neck dissection. Proc Am Soc Clin Oncol. 2003;22: Abstract 881 .

91. Dean N. Wound healing complications with cetuximab therapy. Otolaryngal Head Neck Surg. 2009;141:61.

92. Barnes CJ, Ohshiro K, Rayala SK, El-Naggar AK, Kumar R. Insulin-like growth factor receptor as a therapeutic target in head and neck cancer. Clin Cancer Res. 2007;13:4291-4299.

93. Yu Z, Weinberger PM, Sasaki C, et al. Phosphorylation of Akt (Ser473) predicts poor clinical outcome in oropharyngeal squamous cell cancer. Cancer Epidemiol Biomarkers Prev. 2007;16:553-558.

94. Amornphimoltham P, Patel V, Sodhi A, et al. Mammalian target of rapamycin, a molecular target in squamous cell carcinomas of the head and neck. Cancer Res. 2005;65:9953-9961.
95. Zhang Q, Thomas SM, Xi S, et al. SRC family kinases mediate epidermal growth factor receptor ligand cleavage, proliferation, and invasion of head and neck cancer cells. Cancer Res. 2004;64:6166-6173.

96. Xi S, Zhang Q, Dyer KF, et al. Src kinases mediate STAT growth pathways in squamous cell carcinoma of the head and neck. $J$ Biol Chem. 2003;278:31574-31583.

97. Koppikar P, Choi SH, Egloff AM, et al. Combined inhibition of c-Src and epidermal growth factor receptor abrogates growth and invasion of head and neck squamous cell carcinoma. Clin Cancer Res. 2008;14:4284-4291.

98. Kies M, Ghebremichael M, Katz T, Herbst R, Youssoufian H, Burtness B. EGFR expression by immunohistochemistry (IHC) and response to chemotherapy and cetuximab in squamous cell carcinoma of the head and neck. J Clin Oncol. 2007; P I. Vol 25, No. 18S(June 20 suppl):abstr 6024.

99. Chung C, Ely K, McGarvan L. Increased epidermal growth factor gene copy number is associated with poor prognosis in head and neck squamous cell carcinomas. J Clin Oncol. 2006;24:4170-4176.

100. Licitra L, Rollan F, Bokemeyer C. Biomarker potential of EGFR gene copy number by FISH in the phase III EXTREME study: Platinumbased CT plus cetuximab in first-line R/M SCCHN. J Clin Oncol. 2009;27:15S(suppl):abstr 6005.

101. Chung C, Seeley E, Grigorieva J, Yarbrough W. Mass spectrometry profile as predictor of overall survival benefit after treatment with epidermal growth factor receptor inhibitors in head and neck squamous cell carcinoma. J Clin Oncol. 2009;27:15S(suppl):abstr 6000.

102. Shin DM, Donato NJ, Perez-Soler R, et al. Epidermal growth factor receptor-targeted therapy with $\mathrm{C} 225$ and cisplatin in patients with head and neck cancer. Clin Cancer Res. 2001;7:1204-1213.

103. Worden FP, Kumar B, Lee JS, et al. Chemoselection as a strategy for organ preservation in advanced oropharynx cancer: response and survival positively associated with HPV16 copy number. J Clin Oncol. 2008;26:3138-3146.

104. Kumar B, Cordell KG, Lee JS, et al. EGFR, p16, HPV Titer, Bcl-xL and $\mathrm{p} 53$, sex, and smoking as indicators of response to therapy and survival in oropharyngeal cancer. J Clin Oncol. 2008;26:3128-3137.

105. Psyrri A, Gouveris P, Vermorken J. Human papillomavirus-related head and neck tumors: clinical and research implication. Curr Opin Oncol. 2009;21:201-205.
Biologics: Targets \& Therapy

\section{Publish your work in this journal}

Biologics: Targets \& Therapy is an international, peer-reviewed journal focusing on the patho-physiological rationale for and clinical application of Biologic agents in the management of autoimmune diseases, cancers or other pathologies where a molecular target can be identified. This journal is indexed on PubMed Central, CAS, EMBase, Scopus

\section{Dovepress}

and the Elsevier Bibliographic databases. The manuscript management system is completely online and includes a very quick and fair peerreview system, which is all easy to use. Visit http://www.dovepress. $\mathrm{com} /$ testimonials.php to read real quotes from published authors. 